\title{
Uso medicinal de la marihuana: la discusión pendiente - Réplica
}

\section{Medicinal use of marijuana: the pending discussion - Reply}

Correspondencia Martín Nizama-Valladolid martinnizamav@hotmail.com

Recibido: 11/12/2017 Aprobado: 13/12/2017

Citar como: NizamaValladolid M. Uso medicinal de la marihuana: la discusión pendiente - Réplica. Acta Med Peru. 2017;34(4):341-2

\author{
Martín Nizama-Valladolid ${ }^{1,2,3, a, b, b, c, d}$ \\ 1 Dirección Ejecutiva de Investigación, Docencia y Atención Especializada de Adicciones, Instituto Nacional de Salud Mental \\ Honorio Delgado- Hideyo Noguchi. Lima, Perú. \\ 2 Facultad de Medicina Alberto Hurtado, Universidad Peruana Cayetano Heredia. Lima, Perú. \\ 3 Facultad de Medicina de San Fernando, Universidad Nacional Mayor de San Marcos. Lima, Perú \\ a Médico Psiquiatra; ${ }^{b}$ Médico especialista en Adicciones; ${ }^{c}$ Doctor en medicina; ${ }^{d}$ Docente
}

El artículo sobre la desmitificación del uso medicinal de la marihuana presenta una clara posición contra su legalización con argumentos médicos y sociales que lo avalan, se fundamenta en la experticia del autor y siendo un artículo de sección especial, no es la intención del autor en esta oportunidad, presentar una investigación original lo cual si requeriría de una rigurosidad sistemática.

En el mismo se resalta que aún es insuficiente la evaluación científica de la marihuana sobre sus efectos con fines terapéuticos. Sin embargo se exponen los aspectos positivos de su uso médico respecto a medicamentos derivados de dos cannabinoides: delta 9-tetrahidrocannabilol (dronabinol) y cannabinol (nabilona). Por ejemplo, el uso del dronabinol en la estimulación del apetito en pacientes oncológicos y con $\mathrm{VIH}^{[1]}$.

El uso de la nabilona, aprobado por la FDA, está aconsejado en el manejo sintomático de las náuseas y vómitos que son consecuencia de quimioterapia; igualmente, en el tratamiento de la anorexia en el VIH/SIDA ${ }^{[2]}$. También, se refiere el uso de marihuana, por vía inhalada, con evidencia de buena respuesta en pacientes con dolor neuropático y dolor crónico ${ }^{[3]}$.

Cabe resaltar entonces, que la marihuana como tal, no ha sido aprobada como droga segura y eficaz para ninguna indicación médica por la Federal Drug Administration (FDA) ${ }^{[4]}$. Los medicamentos derivados de los cannabinoides (dronabinol y nabilona) están indicados como paliativos de la emesis, crisis convulsivas y dolor neuropático crónico basado en estudios aleatorizados ${ }^{[5]}$.

En revisiones sistemáticas de ensayos controlados aleatorizados y estudios prospectivos observacionales a largo plazo de los últimos ocho años se evidencia que no hay pruebas suficientes de ningún beneficio de los cannabinoides (dronabinol, nabinol, marihuana medicinal, o tetrahidrocannabinol/cannabidiol en aerosol) para tratar el dolor en el cáncer, el dolor de origen reumático y gastrointestinal, así como la anorexia en el cáncer o el SIDA ${ }^{[6,7]}$. Estas investigaciones no han evaluado los efectos dañinos a largo plazo ${ }^{[5]}$. Además, su uso podría resultar adictivo como lo es en el caso del dronabinol ${ }^{[8]}$.

El uso de marihuana también se ha asociado a múltiples efectos adversos, algunos de los cuales han sido determinados en investigaciones con un alto nivel de confianza ${ }^{[2]}$; como ocurre con la adicción cuando se hace un abuso de la sustancia. 
Otros efectos adversos, respaldados por investigaciones de confianza moderada, fueron desarrollo anormal cerebral, progresión hacia el uso de otras sustancias ilícitas, el desencadenamiento o exacerbación de los síntomas en la esquizofrenia, depresión o ansiedad.

Financiamiento: Autofinanciado.

Conflicto de intereses: Ninguno.

\section{REFERENCIAS BIBLIOGRÁFICAS}

1. DeJesus E, Rodwick BM, Bowers D, Cohen CJ, Pearce D. Use of dronabinol improves appetite and reverses weight loss in HIV/ AIDS-infected patients. J Int Assoc Physicians AIDS Care (Chic). 2007;6(2):95-100

2. Volkow ND, Compton WM, Weiss SR. Adverse health effects of marijuana use. N Engl J Med. 2014;371(9):879.
3. Hancox RJ, Shin HH, Gray AR, Poulton R, Sears MR. Effects of quitting cannabis on respiratory symptoms. Eur Respir J. 2015;46(1):80-7.

4. Food and Drug Administration [Internet]. Hampton, Virginia: FDA; c2017 [citado el 14 de febrero de 2017]. Disponible en: https:// www.fda.gov/

5. Levin FR, Mariani JJ, Pavlicova M, Brooks D, Glass A, Mahony A, et al. Dronabinol and lofexidine for cannabis use disorder: a randomized, double-blind, placebo-controlled trial. Drug Alcohol Depend. 2016;159:53-60.

6. Häuser W1, Fitzcharles MA, Radbruch L, Petzke F. Cannabinoids in Pain Management and Palliative Medicine. Dtsch Arztebl Int. 2017;114(38):627-34

7. Häuser W, Petzke F, Fitzcharles MA. Efficacy, tolerability and safety of cannabis-based medicines for chronic pain management - An overview of systematic reviews. 2017 Oct 15. doi: 10.1002/ ejp.1118. [Epub ahead of print]

8. Mack A, Joy J. Marijuana as Medicine? The Science Beyond the Controversy. Washington, DC: National Academies Press; 2000.

\section{Las ediciones anteriores de Acta Médica Peruana están disponibles en:}

